(C) 2021 IEEE

22nd IEEE Workshop on Control and Modeling for Power Electronics - COMPEL 2021

\title{
Nodal Impedance Assessment in DC Power Distribution Networks
}

R. P. Barcelos and D. Dujic

This material is posted here with permission of the IEEE. Such permission of the IEEE does not in any way imply IEEE endorsement of any of EPFL's products or services. Internal or personal use of this material is permitted. However, permission to reprint / republish this material for advertising or promotional purposes or for creating new collective works for resale or redistribution must be obtained from the IEEE by writing to pubs-permissions@ieee. org. By choosing to view this document, you agree to all provisions of the copyright laws protecting it. 


\section{Nodal Impedance Assessment in DC Power Distribution Networks}

\author{
Renan Pillon Barcelos \\ École Polytechnique Fédérale de Lausanne \\ Lausanne, Switzerland
}

\author{
Dražen Dujić \\ École Polytechnique Fédérale de Lausanne \\ Lausanne, Switzerland
}

\begin{abstract}
The evolution of the power electronics based energy sources and the dc transformer technology are enabling more complex and larger de power distribution systems. For such a system, identifying the impedance characteristic of a certain node is an essential step to investigate the system's stability and expansion capability. To address the challenges of multiconverter connections and bidirectional nodes, this paper investigates a method to approach dc power systems by computing a harmonic solution using the response for multiple degrees of freedom to determine the nodal impedances. It enables a scalable and straightforward procedure to compute each node impedance regardless of the system configuration. The solution is demonstrated for six nodes system with one dc transformer and it can be extended for any number of nodes and de transformers. Theoretical developments are validated by means of simulations and the steady-state and impedance identification process is described in detail.
\end{abstract}

Index Terms-dc power system, DCT, impedance, modal analysis, multi-converters, stability.

\section{INTRODUCTION}

The shift towards the dc power distribution networks (PDN), enabled by power electronics technologies, is changing the nature of the electrical power systems. Nowadays, dc PDN can effectively support energy transformation with high penetration of distributed energy resources and energy storage integration (both increasingly being $\mathrm{dc}$ by nature) in future systems. In applications, such as distribution networks in marine vessels, dc has already showed a proven power distribution system with elevated savings compared to state-of-the-art ac systems. [1].

In dc PDNs, the inclusion of the dc transformer (DCT) to the dc power system, creates a special dc system layout, where the DCT links different dc power sources and loads in one integrated system. Thus, it allows the power exchange between two different dc buses, sharing loads and sources in an interconnected PDN. In this context, the DCT is an isolated dcdc power converter acting as a power transformer between two dc lines, operating in an open-loop [2], meaning that it does not receive voltage or power set point. Yet, as a bidirectional element, the DCT is capable of following the natural power flow [3]. However, although dc-dc converters are a mature technology, more research is still needed to understand its high voltage and high power promises in dc PDN applications.

Therefore, the dc PDN can be formed by some type of ac/dc power converters aiming to supply the dc voltage and feed loads; or, by renewable generation with power converter energy processing, where the source aims to inject its maximum power generation to the grid, feeding loads and energy storage units. Thus, the interaction between different types of sources and different control strategies can bring extra challenges in terms of power management.

Yet, in dc power systems, the power-electronics-driven loads are seen as a constant power load (CPL), that has a negative incremental impedance behavior [4]. The CPL characteristic reduces the effective damping of the system and may induce destabilizing effects into the system [5]. Hence, there is a need to understand the system level behavior when different converters are connected to the dc PDN and the line impedance needs to be taken into account as well for the connection path (DCT connection)

Some of the existing solutions to analyse the dc system stability simplify the connection point, turning the flow between nodes totally ruled by the sources, and in this way, enabling the parallel equivalent of several converters and loads [6][9]. This approach is applicable and well justified when all the converters forming the $\mathrm{dc}$ grid have the same dynamic behavior. However, when relating to systems with different characteristics, ac/dc converters from different manufactures (different controllers, different frequency), bidirectional nodes, different transmission line sizes, and not negligible impedance in the connection point, this approaches is not suitable anymore.

Other works are addressing these problems using harmonic resonance analysis [10]-[12]. This approach is able to tackle these problems and use modal analysis to compute the system response. However, identifying the system's resonance is not enough to have a concrete parameter and identify the system stability. Nevertheless, as there is no angle difference between the nodes (the power flow is given by the voltage difference), and the system's dynamics are fully ruled by the voltage dynamic, it is possible to find a solution for the nodes' voltages in order to identify the system behavior to an external excitation response.

Therefore, this paper addresses dc power systems with a multiple degrees of freedom solution for the voltage dynamic, identifying the voltage behavior for each node and computing the impedance characteristic of the system. This approach allows us to perform the following analysis: dc system planning, operation, network expansion studies, damping provision and 


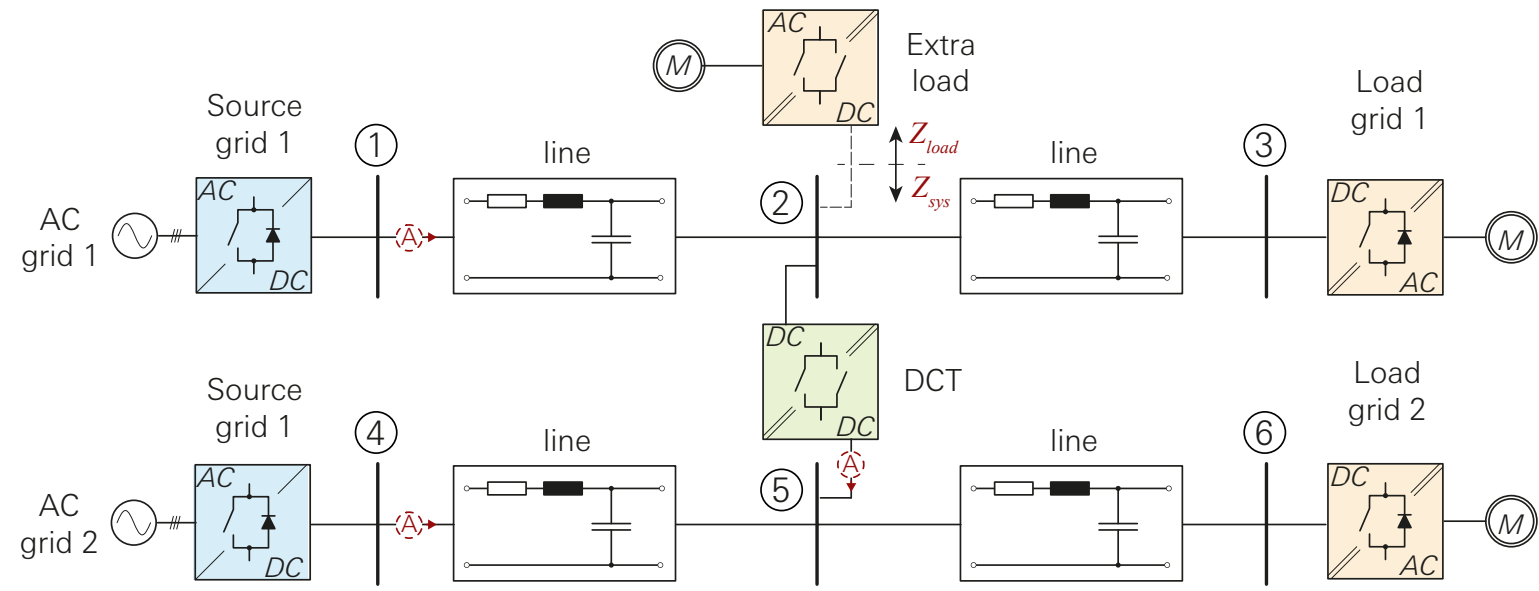

Fig. 1. De power system with six nodes and one LLC-DCT. An extra element to be added to the system.

stability verification.

The following sections will show the procedure to find the multiple degrees of freedom response of the voltage for an external excitation, the dc power system models description, and a detailed solution with an example for a six nodes and one DCT. At the end, the change in the system's characteristics when a new power converter is added to the grid is analyzed.

\section{RESPONSE FOR MULTIPLE DEGREES OF FREEDOM}

Electrical systems have (especially dc electrical systems) the dynamic behavior highly related to properties of the voltage in the system. In this way, let us consider the voltage description on a generic element for a current excitation:

$$
Q \ddot{v}+C \dot{v}+Y v=I,
$$

where $\mathrm{Q}$ is the charge matrix, $\mathrm{C}$ is a capacitance matrix, $\mathrm{Y}$ admittance matrix, and $\mathrm{I}$ is the urrent external force. The second derivative of the voltage is out of scope of this work.

Therefore, let us assume the solution in the form of $v=$ $V e^{s t}$, then replacing it in (1), with $v(0)=0$, implies

$$
C s V e^{s t}+Y V e^{s t}=I e^{s t},
$$

which implies that,

$$
V=\underbrace{[C s+Y]^{-1}}_{Z} I .
$$

Thus, $Z$ is called the impedance matrix of the system and each element of this matrix represents a transfer function. Each transfer function gives the response of the voltage for an excitation in current. In addition to that, the control loop is another dynamic relating the voltage and current present in the system, where the control loop is described by,

$$
\frac{\Delta i_{d c}}{\Delta v_{d c}}=f(s)=T \text {. }
$$

Thus, the final transfer function matrix is:

$$
V=\underbrace{[C s+Y+T]^{-1}}_{H_{z}} I .
$$

Although the control loops in the equivalent models derived in the next section are already in the form of admittance and can easily be added in the $Y$ matrix, adding the control loops as an external matrix $T$ allows us to use the nodal admittance as the $Y$ matrix. Therefore, $Y$ will always describe the system configuration, $T$ will depend on the control strategy available at the node, and $C$ represents the node capacitance value.

\section{METHODOLOGY}

Fig. 1 shows an example of dc power system with six nodes and one DCT connecting two different voltage dc grids. It is important to note that the DCT connections are simple connection nodes with no voltage or current being regulated directly.

The solution to analyze this system can be addressed separately in two steps. Firstly, the steady-state solution for the load flow calculation, and secondly, in the frequency domain for the resonance analysis. The load flow solution will provide information about the system operating point. Every time the load changes, a new operating point is defined. Thus, it is crucial to check the steady-state solution every time there is a change in the dc PDN to ensure the system's operability.

The frequency analysis will provide crucial information about the system characteristics. It will show each node's impedance characteristic; therefore, the critical resonances and their damping will be highlighted.

\section{A. Part I - Steady-state solution}

Usual solutions to power flow in dc systems are supported by an external ac power flow loop [13], [14], which is not present in a purely dc power system. Thus, as there is no inductance/capacitance, neither angle difference at steady state in dc system, it is impossible to compute the source's power injection to the system using a nodal approach, without adding 
some extra equations. One option is to use the Modified Mode Analysis (MNA) [15] to find a nodal solution. This formulation allows us to include extra node/branch equation to compute the voltages/currents of the system. It can also include non-linearity effects (e.g., CPL), however, it will require the use of iterative methods, which in a purely dc-system is not necessary.

Therefore, the steady state solution is reached through the following steps:

1) Determine the Nodal Admittance

$$
I=Y_{\text {nodal }} V
$$

where, $I$ is the node current, $V$ the node voltage and $Y_{\text {nodal }}$ the Nodal Admittance.

2) Describe the Modified Nodal Analysis matrix

$$
\underbrace{\left[\begin{array}{ll}
Y_{\text {nodal }} & B \\
C & D
\end{array}\right]}_{A} \underbrace{\left[\begin{array}{c}
V \\
I
\end{array}\right]}_{x}=\underbrace{\left[\begin{array}{l}
J \\
F
\end{array}\right]}_{b},
$$

where the vectors $J$ and $F$ are the excitation, $Y_{\text {nodal }}$ Nodal Admittance, $B$ contain the Kirchhoff current equations with respect to the additional current, $C$ and $D$ are the branch constructive relations, differentiated with respect to the unknown vector.

3) Solve linear system of (7)

$$
x=A \backslash b
$$

After finding the current and voltages, the current flow and power injection can easily be found for each node, and therefore, the operating system point can be identified.

\section{B. Part II - Frequency domain solution}

The second step is finding an analytical solution to identify the driven point of the system. Using the nodal information, which allows us to extend the method to larger systems, the nodal admittance and the response for multiple degrees of freedom of the voltage are described. With this approach, the nodal impedance is easily extracted and the frequency characteristic for the node impedance is calculated for any node of the system.

The following steps describe the procedure to promote such analysis:

1) Determine the Nodal Admittance

$$
I=Y_{\text {nodal }} V
$$

2) Compute the matrix $C$ and $T$ of (3) and (4),

$$
\begin{gathered}
C=\operatorname{diag}\left(c_{1}, c_{2}, \ldots, c_{i}\right) \quad i=1,2, \ldots, n \\
T=\operatorname{diag}\left(Y_{s / l, 1}, Y_{s / l, 2}, \ldots, Y_{s / l, i}\right) \quad i=1,2, \ldots, n
\end{gathered}
$$

where, $n$ is the number of nodes and $s / l$ is source or load.

3) Find the impedance's transfer function according to (5):

$$
V=H_{z} I
$$

where $V$ is the node's voltage, $H_{z}$ the impedance transfer function and $I$ the node's current.

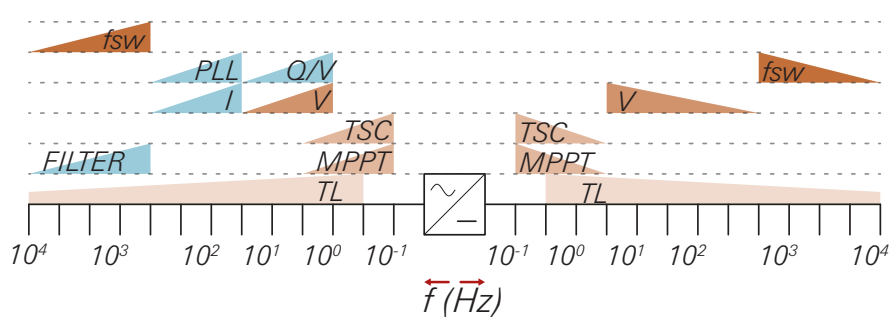

Fig. 2. Usual controls bandwidths present in dc and ac power converter based systems. Frequency axis not to be scaled. Blue color highlights the extra elements. Equivalent brown color highlight similar functions. y-axis represents the PE designer degrees of freedom to change its frequency impact (top - more flexible). The triangle states for the beginning of its impact and attenuation for higher frequencies.

\section{MODEL DESCRIPTION}

To derive a proper model, it is essential to to correctly identify the desired frequency range of analysis. Fig. 2 shows the control strategies and the usual resonant frequencies present for the ac (left side) and dc (right side) power electronics based energy system.

This figure is purely illustrative, however, it helps the reader to understand the frequency range for analysis. It shows the resonances in passive elements (Transmission line - TL and Filters), power source controllers (Maximum power point tracking - MPPT and Turbine speed control - TSC), grid controllers (Voltage and current control, power droops, phase locked loop - PLL), and the power converter switching frequency.

For a dc PDN, the voltage control bandwidth of dc system is usually set around $10 \mathrm{~Hz}$ to almost $1 \mathrm{kHz}$. Therefore, as it is desirable to have the system description within the voltage control bandwidth, the model should be valid for this frequency range. Another resonance that impacts this range of frequency is the transmission line resonance, and special care should be given to this model.

\section{A. Transmission line}

Lines and cables play an important role in harmonic resonances. Depending on the range of frequency and the length, the effects of long-line become critical. In this paper, a buried medium voltage cable with the data in Table I is considered. Thus, Fig. 3 shows the frequency response for different cable models; i) using distributed parameters and ii) one section $\pi$ equivalent model. Two different cable lengths of $20 \mathrm{~km}$ and $50 \mathrm{~km}$ are simulated to demonstrate the model difference. As stated in this figure, the considered cable can be faithfully represented by the one section $\pi$-equivalent model for the 20 $\mathrm{km}$ cable length until $1 \mathrm{kHz}$, and it is also expected for short cables. However, if the line of $50 \mathrm{~km}$ length is considered, one section $\pi$-equivalent model does not properly capture the frequency response above $500 \mathrm{~Hz}$. Then, more $\pi$-sections or a frequency-dependent model should be used to faithfully capture the frequency response for long cables. 


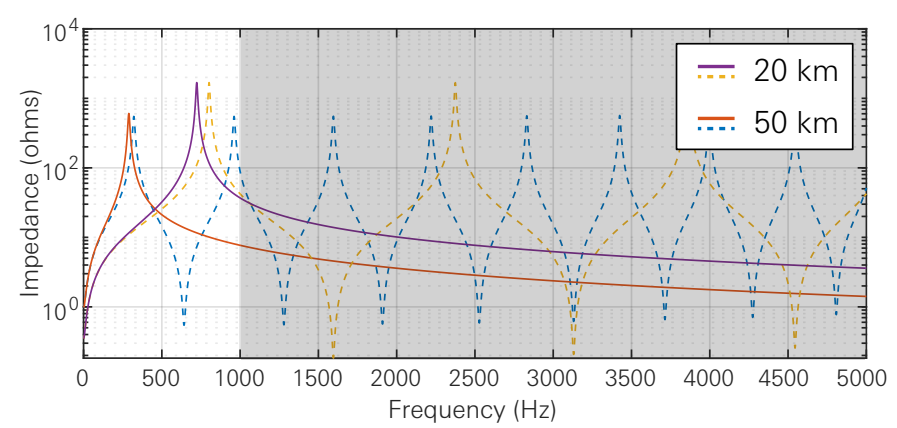

Fig. 3. Comparison of the distributed parameters (dashed) and one section $\pi$-lumped (solid line) model, for the medium voltage cable with $20 \mathrm{~km}$ and $50 \mathrm{~km}$ length long. Shaded part shows the frequency out of the scope.

TABLE I

BURIED MEDIUM VOLTAGE CABLE DATA [16].

\begin{tabular}{lcc}
\hline & Value & Unit \\
Nominal current & 1 & $\mathrm{kA}$ \\
Voltage & 11 & $\mathrm{kV}$ \\
Resistance & 17.6 & $\mathrm{~m} \Omega / \mathrm{km}$ \\
Inductance & 0.268 & $\mathrm{mH} / \mathrm{km}$ \\
Capacitance & 0.904 & $\mu \mathrm{F} / \mathrm{km}$ \\
\hline
\end{tabular}

Therefore, using the cable described in Table I, this methodology can be used for lines until $20 \mathrm{~km}$ of length, using one section $\pi$-equivalent model, being valid until $1 \mathrm{kHz}$.

\section{B. Sources and Loads}

Fig. 4 shows the equivalent circuits for the sources and load devices. The sources considered in the work are all ac/dc converters with closed loop controlled voltage. Thus, to allow further impedance-based stability studies, it is convenient to draw an equivalent circuit following [17]. Considering that the slower outer voltage control, determines its behavior in the frequency domain, and a PI voltage controller, the ac/dc converter can be represented as in Fig. 4 (b), with the source admittance value of

$$
Y_{s}=\frac{s K_{p}+K_{i}}{s} \times K_{G_{v i}}
$$

where, $K_{p}$ and $K_{i}$ are the proportional and integration gain, respectively, and $K_{G_{v i}}$ is the inner control loop compensation gain, usually determined by $K_{G_{v i}}=3 V_{d} / 2 V_{d c}$.

The loads behave as CPLs within their current control loops bandwidth [5]. Therefore, for a certain operation point $V_{o}$, the linearized model can be represented as Fig. 4 (d), where the load admittance value is:

$$
Y_{l}=-\frac{V_{o}^{2}}{P_{c l p}},
$$

and

$$
I_{c l p}=2 \frac{P_{c l p}}{V_{o}}
$$

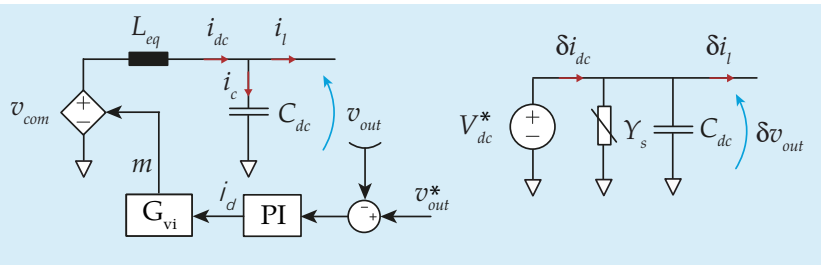

(a)

(b)

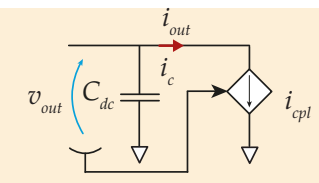

(c)

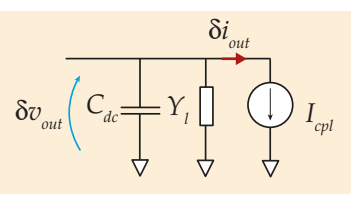

(d)
Fig. 4. Equivalent linear models. (a) ac/dc converter dynamic equivalent model; (b) ac/dc converter linearized model; (c) load dynamic equivalent model; and (d) load linearized model.

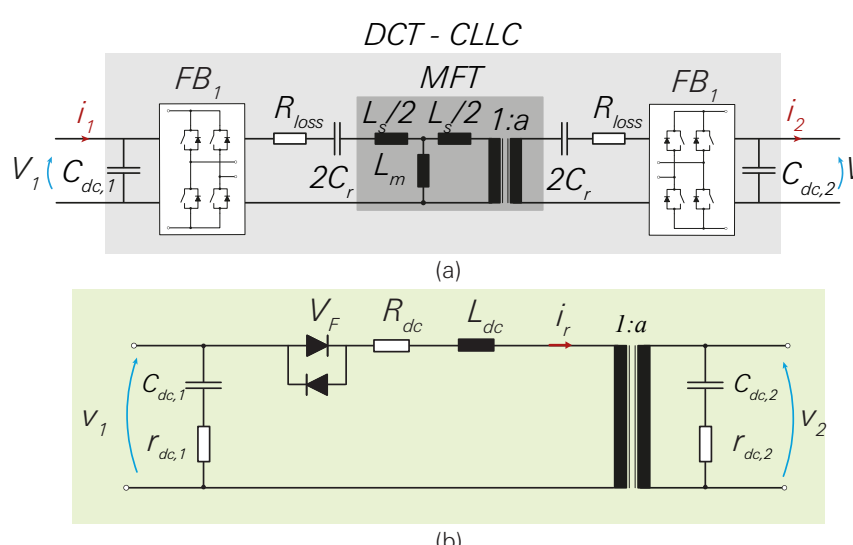

Fig. 5. DC transformer topology. (a) FB bidirectional LLC (b) DC transformer dynamic equivalent model.

\section{DCT - LLC transformer model}

The DCT used in this work is shown in Fig. 5 (a), it is a full bridge (FB) bidirectional LLC, with split resonant tank on each side of the medium frequency transformer (MFT) [2]. The dynamic equivalent model is shown in Fig. 5 (b) [18]. $V_{F}$ represents the total losses on the power semiconductors. $R_{d c}$ represents the losses caused by the total series resistance in the current path, it can be calculated by:

$$
R_{\mathrm{dc}}=\beta^{2} R_{\mathrm{total}}+\left(\beta^{2}-1\right)\left(r_{c 1}+r_{c 2}^{\prime}\right),
$$

where $R_{\text {total }}$ is the sum of all series resistances in the current path. The energy stored in the resonant tank is represented by $L_{d c}$, calculated by (17).

$$
L_{\mathrm{dc}}=\alpha^{2} L_{s}
$$

For the case of piecewise sinusoidal current (i.e. $f_{s}=f_{o}$ ), $\alpha$ and $\beta$ can be found 
TABLE II

PARAMETERS OF THE TESTED SYSTEM.

\begin{tabular}{ccc}
\hline & Value & Unit \\
\hline Rated Power & 10 & $\mathrm{MW}$ \\
Source grid 1 & 6 & $\mathrm{kV}$ \\
Source grid 2 & 12 & $\mathrm{kV}$ \\
Load grid 1 & 866.67 & $\mathrm{~A}$ \\
Load grid 2 & 433.33 & $\mathrm{~A}$ \\
Extra Load & 433.33 & $\mathrm{~A}$ \\
$z_{t l}$ & $0.0176+s 0.268 \times 10^{-3}$ & $\Omega / \mathrm{km}$ \\
$z_{d c t}$ & $0.014+s 39.97 \times 10^{-6}$ & $\Omega$ \\
$c_{D C T}$ & 8 & $\mathrm{mF}$ \\
$1: \mathrm{n}$ & $1: 2$ & - \\
$c_{d c}$ & 2.5 & $\mathrm{mF}$ \\
$K_{p}$ & 0.125 & $1 / \Omega$ \\
$K_{i}$ & 0.05 & $1 / \Omega$ \\
\hline
\end{tabular}

$$
\alpha=\frac{\pi}{2} \frac{f_{0}}{f_{\mathrm{s}}} \quad \text { and } \quad \beta=\frac{\pi}{2 \sqrt{2}} \sqrt{\frac{f_{0}}{f_{\mathrm{s}}}},
$$

otherwise the coeficients are calculated by:

$$
\alpha:=\frac{\hat{i}_{\mathrm{R}}}{\overline{\bar{i}}_{\mathrm{R}}} \text { and } \beta:=\frac{\tilde{i}_{\mathrm{R}}}{\bar{i}_{\mathrm{R}}},
$$

where, $\tilde{i}_{\mathrm{R}}$ is the rms current of the switched converter, $\bar{i}_{\mathrm{R}}$ is the local average current and $\hat{i}_{\mathrm{R}}$ is related to the storage energy in the MFT [18].

\section{Simulation RESUlts}

The system of Fig. 1 with the data showed in Table II was simulated to demonstrate the method, firstly without the extra load being the part of the system. The ac grid is modeled as an ideal voltage source and a series impedance of $z_{s}=0.001+j 0.01$ (p.u.), and each transmission line has $10 \mathrm{~km}$ of length.

At first, one can compute the steady-state solution to identify the operation point. The details of the matrices to compute this example are available in the appendix. Table III shows the steady-state solution comparing the part I methodology computed in Matlab and the switched model simulated in PLECS. It shows the voltage and current of the system, with a good matching of results. The current direction is stated in Fig. 1, and the power sources' currents and the DCT voltages are shown in Fig. 6. The oscillations in this figure are the bus voltage oscillations regulated by the power converters.

For the frequency analysis, the system was computed using (12), and compared with the impedance measurement in PLECS. The impedance measurement was performed by injecting a multi-tone signal to the node and measuring the frequency response on the same node. Fig. 7 illustrates how the signal injection is performed for any power converter and for the dc PDN node. The signal was generated by a 12-bitlength shift register resulting in a 4095-bit-long PRBS signal [19]. Therefore, after recording the current and voltage of interest, taking the DFT, and plotting it in the Bode diagram, one can find the frequency characteristic of the impedance.

TABLE III

STEADY STATE SOLUTION

\begin{tabular}{lccc}
\hline Voltage & Calculated & Simulated & Unit \\
\hline Node 1 & 6 & 6 & $\mathrm{kV}$ \\
Node 2 & 5.937 & 5.936 & $\mathrm{kV}$ \\
Node 3 & 5.784 & 5.783 & $\mathrm{kV}$ \\
Node 4 & 12 & 12 & $\mathrm{kV}$ \\
Node 5 & 11.879 & 11.878 & $\mathrm{kV}$ \\
Node 6 & 11.8032 & 11.803 & $\mathrm{kV}$ \\
\hline Current & Calculated & Simulated & Unit \\
\hline Node 1 & 358.22 & 360.39 & $\mathrm{~A}$ \\
Node 4 & 687.55 & 687.67 & $\mathrm{~A}$ \\
DCT & -254.22 & -254.30 & $\mathrm{~A}$ \\
\hline
\end{tabular}
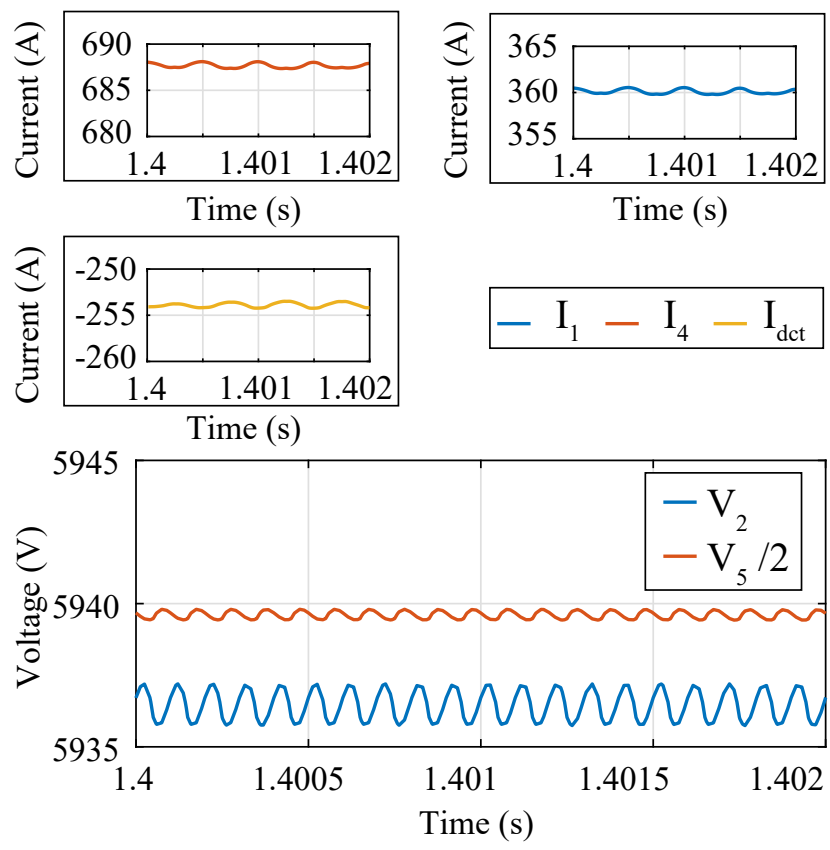

Fig. 6. Simulation results of Node 1, Node 4 and DCT current on the top. Node 2 and Node 5 voltages on the bottom.

The same procedure is used for equation (12), where the signal $I$ is injected in a certain node and the voltage $V$ is read in another/or the same node, creating the impedance $Z_{x y}$, meaning injection in node $x$ and measurement in node $y$. The capacitor $C_{2}$ in Fig. 7 represents the equivalent capacitance of the node, wherein this case is the sum of the DCT capacitor and the contribution of the one section $\pi$-equivalent of the transmission line.

Fig. 8 shows the node's impedance for the Nodes 2, 3, 5, and 6, using equation (12) and the impedance measurement in PLECS, without the extra load.

The method was able to faithfully identify the system's resonances and follow the impedance magnitude within the frequency range of interest. The mismatch around $200 \mathrm{~Hz}$ and $300 \mathrm{~Hz}$ is due to the DCT model, whose equivalent circuit does not map all the passive elements. Yet, it is interesting to note (comparing $Z 22$ and $Z 55$ ) that the lower voltage side 


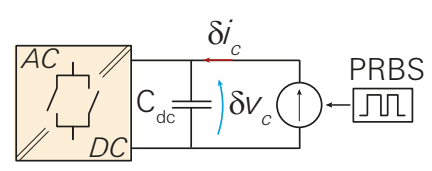

(a)

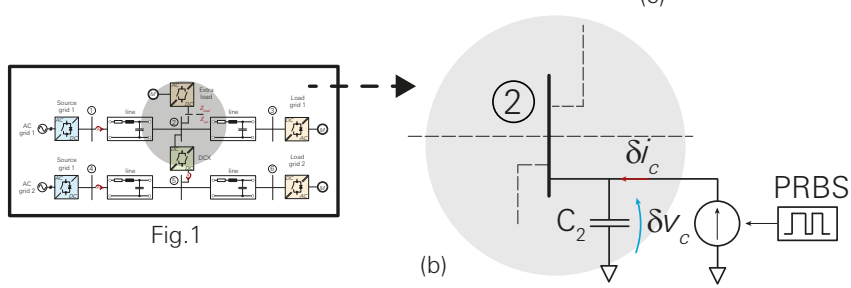

Fig. 7. Impedance measurement procedure. (a) Power converter, (b) PDN node and (c) the bode plot process.

has its resonances less damped than the higher voltage side.

Identifying the nodes' impedance is an essential step to understanding the whole system behavior in frequency domain. With the information provided by Fig. 8 one can identify the nodes that have features closely to sources or loads behavior, the critical resonance frequencies, and the impact of the voltage level on the impedance characteristic, for example. Besides that, from Fig. 8, one can say that the dc PDN has a certain impedance at the connection point and study the possibility to add new elements to the grid.

\section{A. Addition of an extra power converter}

To evaluate the system response when adding a new element, one can include an extra load converter to Node 2. The first important consideration is performing the MNA for the load flow solution to ensure that the two power sources can supply the new load. Through Table II and III, we see that the system is previously operating with half of the power, where each load consists of $0.5 \mathrm{pu}$, and Node 1 feeds $0.2 \mathrm{pu}$ and Node 4 with $0.8 \mathrm{pu}$. Therefore, adding the new power converter in Node 2 and computing the MNA leads Node 1 to feed $0.5 \mathrm{pu}$ and Node $41 \mathrm{pu}$ of current. Thus, this new load can be added to the system.

Fig. 9 shows the initial impedance characteristic of the connection node before adding the new load and the input impedance characteristic of the new converter tested individually $\left(Z_{A F E}\right)$. The input impedance was extracted by injecting the PRBS signal to the input port and measuring the frequency response.

Therefore, after connecting the converter to the grid, a new system is formed, and the new impedance behavior at this node is shown in Fig. 10. Using the same methodology, the impedance response using (12) and the impedance measurement injecting a PRBS signal showed similar results, highlighting a new resonance frequency around 40-60 Hz and more damping around $70 \mathrm{~Hz}$.

By adding this new power converter to the dc PDN, Node 4 operates at its maximum power, while Node 1 is only half of its capacity. The new converter created a completely new
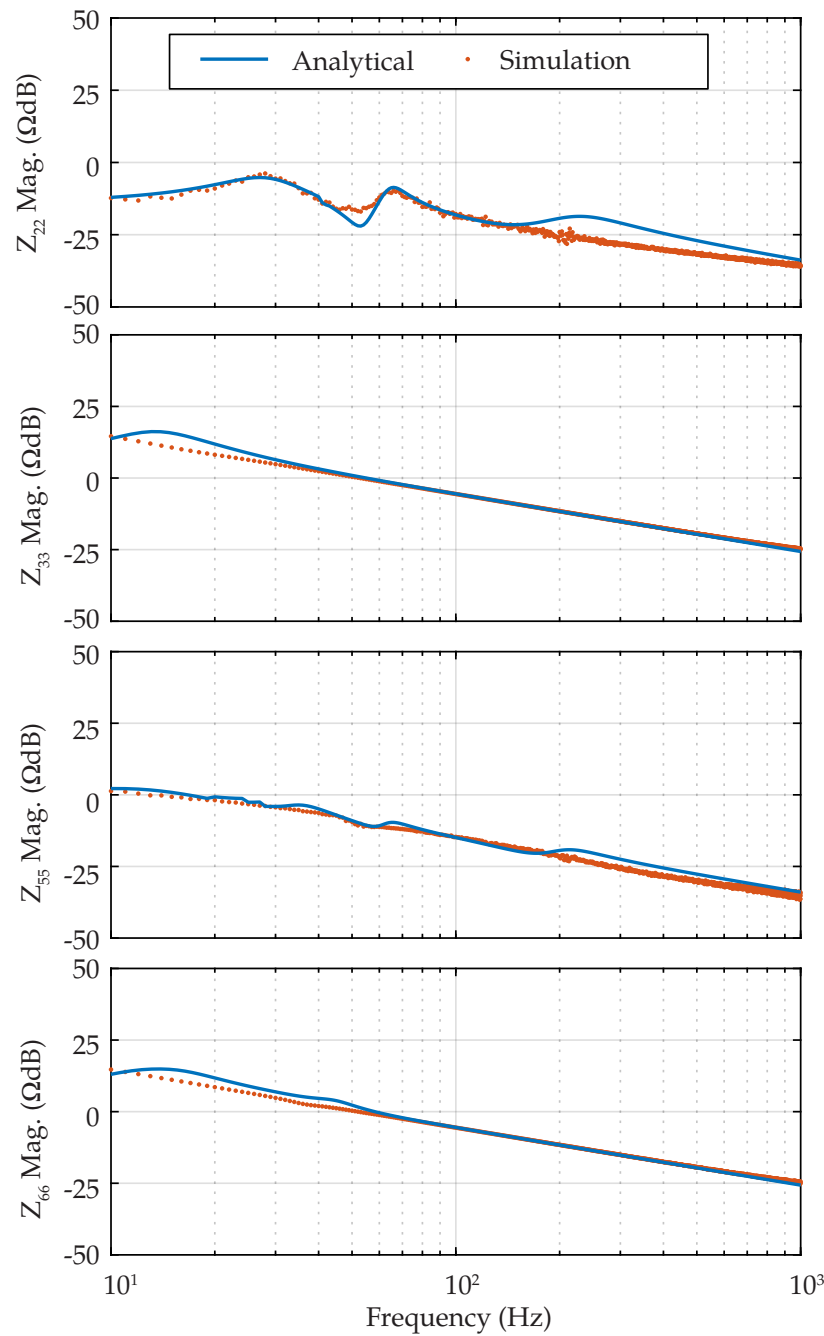

Fig. 8. Impedance characteristic of nodes 2, 3, 5, 6, using equation (12) and impedance measurement from the switched system in PLECS, without extra load in node 2 .

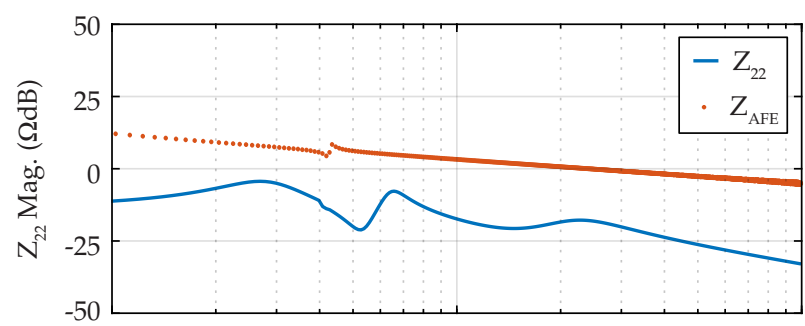

Fig. 9. Impedance characteristic of node 2 and the extra power converter admittance characteristic. 

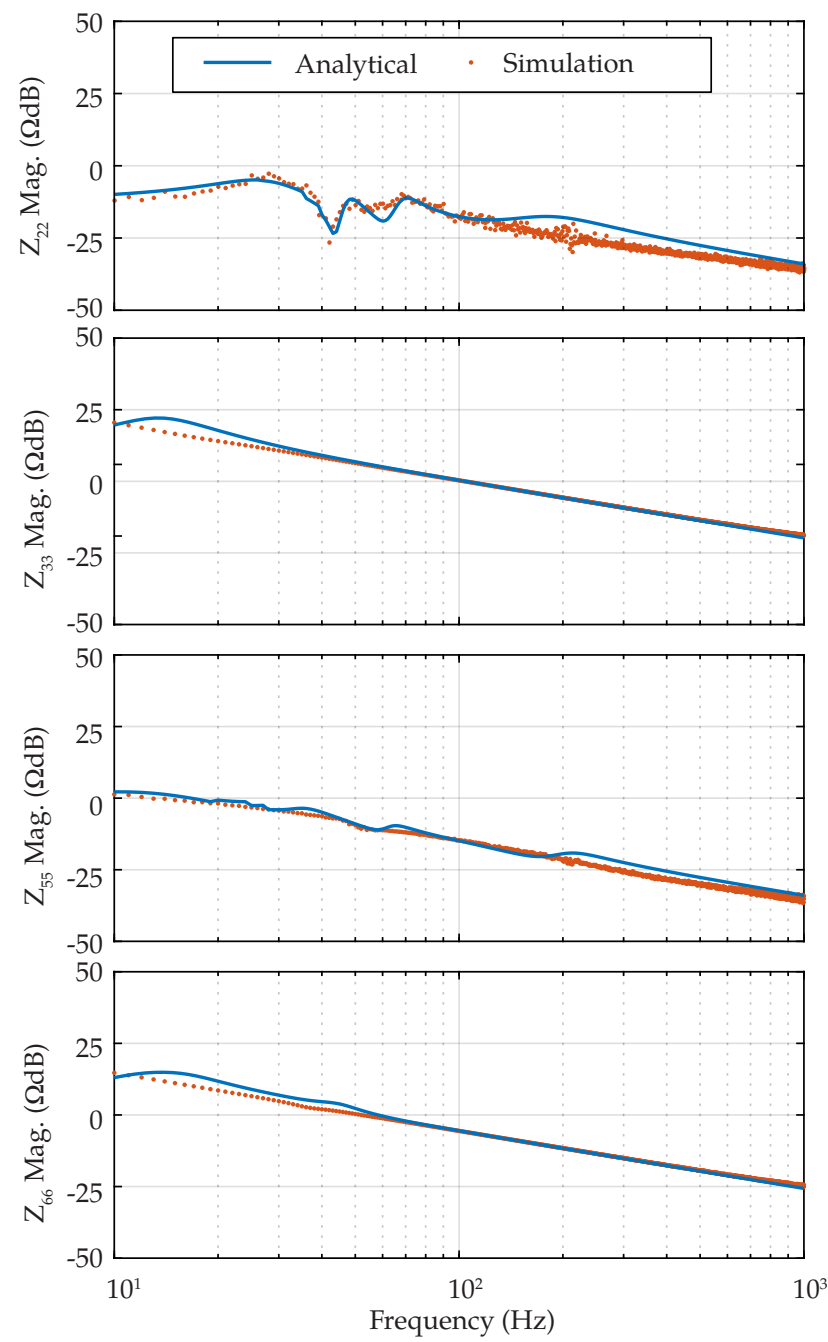

Fig. 10. Impedance characteristic of nodes 2, 3, 5, 6, using equation (12) and impedance measurement from the switched system in PLECS after connecting the new load.

dynamic around 40 and $70 \mathrm{~Hz}$ in terms of the frequency response. However, every resonance is well damped, and similar impedance characteristics resulted from its inclusion. The inclusion of the new load also provoked an increase of the Z33 impedance characteristic in low frequency. Other nodes did not sense the change.

\section{CONCLUSION}

This paper presented a method to compute the node's impedance using the equation of motion for the voltage response and a methodology to compute the operation point of a dc system without knowing the voltage source's power injection - both critical information to perform any analysis in dc power systems.

The solution of part I overcomes the lack of angle and reactive power to calculate the power flow using a nodal approach, allowing the extensibility of the method. Part II of the solution showed the frequency characteristic to find the node's impedance, showing that this approach computes the impedance correctly within the voltage control bandwidth. These solutions can boost the dc power system analysis and allow further investigation in this area.

Further studies with different voltages levels, larger systems, and more aggressive extra elements can be performed to promote dc power system planning and operation analysis. Elements participation factor, node's contribution to the resonances, and its relationship to the nodal impedance, are some of the well-known indicators in the ac power system planning studies that can be extended to the dc power system analysis using the tools addressed in this paper.

\section{APPENDIX}

\section{DESCRIPTION OF MATRICES FOR THE TESTED SYSTEM}

This section describes the matrices for the steady-state and frequency analysis of the system shown in Fig. 1 with data in Table II. The calculation for the Part I is performed in per unit values, and Part II is in SI values. This choice is just for convenience as the switched model in PLECS is computed in SI values. If using per unit values, the correct base should be used to find the impedance value for the desirable side after the calculation.

The element-wise construction was made through the following incidence matrix (element $\backslash$ node):

$$
\mathrm{a}=\left[\begin{array}{cccccc}
1 & -1 & 0 & 0 & 0 & 0 \\
0 & 1 & -1 & 0 & 0 & 0 \\
0 & 1 & 0 & 0 & -1 & 0 \\
0 & 0 & 0 & 1 & -1 & 0 \\
0 & 0 & 0 & 0 & 1 & -1
\end{array}\right]
$$

Therefore, the primitive admittance to build the admittance nodal for the steady-state solution is:

$$
y_{p}=\operatorname{diag}\left(\begin{array}{lllll}
0.0508 & 0.0508 & 0.0014 & 0.0127 & 0.0127
\end{array}\right)^{-1}
$$

and then,

$$
Y_{\text {nodal }}=a^{T} y_{p} a .
$$

The matrices to complete the MNA equation are:

$$
\begin{gathered}
\mathrm{B}^{T}=\left[\begin{array}{cccccc}
-1 & 0 & 0 & 0 & 0 & 0 \\
0 & 0 & 0 & -1 & 0 & 0
\end{array}\right], \\
\mathrm{C}=\left[\begin{array}{llllll}
1 & 0 & 0 & 0 & 0 & 0 \\
0 & 0 & 0 & 1 & 0 & 0
\end{array}\right], \\
\mathrm{D}=\left[\begin{array}{ll}
0 & 0 \\
0 & 0
\end{array}\right],
\end{gathered}
$$

and

$$
\mathrm{J} \mid \mathrm{F}^{T}=\left[\begin{array}{lllllllll}
0 & 0 & -0.5 & 0 & 0 & -0.5 & \mid & 1 & 1
\end{array}\right] .
$$

Therefore, solving (7) for $x$, one can find the voltages of the nodes and the current for the two voltage sources. 
For Part II, the nodal admittance is computed with the same incidence matrix, including the inductance in the primitive admittance. As it is desirable to use the values in SI, it is necessary to reflect all the parameters correctly to the desirable node's side before computing $H_{z}$ in (5). Thus, for the nodes at the lower voltage side, it yields,

$$
y_{p}=\operatorname{diag}\left(10 . z_{t l} \quad 10 . z_{t l} \quad z_{D C T} \quad 10 . z_{t l}^{\prime} \quad 10 . z_{t l}^{\prime}\right)^{-1} .
$$

where $z_{i}^{\prime}=1 / n^{2} . z_{i}$, and the construction of $Y_{\text {nodal }}$ follows equation (22).

The shunt capacitance matrix and control equivalent matrix also need to be reflected. Then,

$$
C=\operatorname{diag}\left(c_{d c} \quad c_{D C T} \quad c_{d c} \quad c_{d c}^{\prime} \quad c_{D C T}^{\prime} \quad c_{d c}^{\prime}\right),
$$

and,

$$
T=\operatorname{diag}\left(Y_{s, 1} \quad 0 \quad Y_{l, 1} \quad Y_{l, 2}^{\prime} \quad 0 \quad Y_{s, 2}^{\prime}\right),
$$

where $c_{i}^{\prime}=n^{2} \cdot c_{i}$ and $Y_{i}^{\prime}=n^{2} \cdot Y_{i}$.

Thus, to select the desirable transfer function from (12), vectors $V$ and $J$ act as pointers, where $J$ is the current injection in a certain node, and $V$ is the resultant voltage of a certain node.

Therefore, selecting for example, node 3, where all the elements of $V$ and $J$ of (12) are zeros, except for the 3rd row that is 1 , we find:

$$
V_{3}=H_{3,3} J_{3},
$$

where $H_{3,3}$, is the impedance transfer function of node 3 .

\section{ACKNOWLEDGMENT}

The results presented in this paper are a part of the EMPOWER project that has received funding from the European Research Council (ERC) under the European Unions Horizon 2020 research and innovation programme (Grant agreement No. 818706).

\section{REFERENCES}

[1] K. Kim, K. Park, G. Roh, and K. Chun, "DC-grid system for ships: a study of benefits and technical considerations," Journal of International Maritime Safety, Environmental Affairs, and Shipping, vol. 2, no. 1, pp. 1-12, nov 2018.

[2] J. Kucka and D. Dujic, "Smooth power direction transition of a bidirectional llc resonant converter for dc transformer applications," IEEE Transactions on Power Electronics, vol. 36, no. 6, pp. 6265-6275, 2021.
[3] — , "Current limiting in overload conditions of an llc-converter-based dc transformer," IEEE Transactions on Power Electronics, vol. 36, no. 9, pp. $10660-10672,2021$

[4] A. Emadi, A. Khaligh, C. Rivetta, and G. Williamson, "Constant power loads and negative impedance instability in automotive systems: definition, modeling, stability, and control of power electronic converters and motor drives," IEEE Transactions on Vehicular Technology, vol. 55, no. 4, pp. $1112-11 \overline{25,2006 .}$

[5] Y. Gui, R. Han, J. M. Guerrero, J. C. Vasquez, B. Wei, and W. Kim "Large-signal stability improvement of dc-dc converters in dc microgrid," IEEE Transactions on Energy Conversion, pp. 1-1, 2021.

[6] S. Sudhoff, S. Glover, P. Lamm, D. Schmucker, and D. Delisle, "Admittance space stability analysis of power electronic systems," IEEE Transactions on Aerospace and Electronic Systems, vol. 36, no. 3, pp. 965-973, 2000.

[7] X. Wang, R. Yao, and F. Rao, "Three-step impedance criterion for smallsignal stability analysis in two-stage dc distributed power systems," IEEE Power Electronics Letters, vol. 1, no. 3, pp. 83-87, 2003.

[8] A. Riccobono and E. Santi, "A novel passivity-based stability criterion (pbsc) for switching converter dc distribution systems," in 2012 Twenty-Seventh Annual IEEE Applied Power Electronics Conference and Exposition (APEC), 2012, pp. 2560-2567.

[9] H. Li, F. Ren, C. Liu, Z. Guo, J. L, B. Zhang, and T. Q. Zheng, "An extended stability analysis method for paralleled dc-dc converters system with considering the periodic disturbance based on floquet theory," IEEE Access, vol. 8, pp. 9023-9036, 2020

[10] L. Hong, W. Shu, J. Wang, and R. Mian, "Harmonic resonance investigation of a multi-inverter grid-connected system using resonance modal analysis," IEEE Transactions on Power Delivery, vol. 34, no. 1, pp. 63-72, 2019.

[11] J. Rommes, N. Martins, and F. D. Freitas, "Computing rightmost eigenvalues for small-signal stability assessment of large-scale power systems," IEEE Transactions on Power Systems, vol. 25, no. 2, pp. 929 938, 2010.

[12] W. Du, Q. Fu, and H. Wang, "Open-loop modal coupling analysis for a multi-input multi-output interconnected mtdc/ac power system," IEEE Transactions on Power Systems, vol. 34, no. 1, pp. 246-256, 2019.

[13] J. Lei, T. An, Z. Du, and Z. Yuan, "A general unified ac/dc power flow algorithm with mtdc," IEEE Transactions on Power Systems, vol. 32, no. 4, pp. 2837-2846, 2017.

[14] C. Liu, B. Zhang, Y. Hou, F. F. Wu, and Y. Liu, "An improved approach for ac-dc power flow calculation with multi-infeed dc systems," IEEE Transactions on Power Systems, vol. 26, no. 2, pp. 862-869, 2011.

[15] C.-W. Ho, A. Ruehli, and P. Brennan, "The modified nodal approach to network analysis," IEEE Transactions on Circuits and Systems, vol. 22, no. 6, pp. 504-509, 1975.

[16] Nexans, "6-36kV Medium Voltage Underground Power Cables," Tech. Rep., 2009.

[17] J. Sun, "Impedance-based stability criterion for grid-connected inverters," IEEE Transactions on Power Electronics, vol. 26, no. 11, pp. 3075$3078,2011$.

[18] J. E. Huber, J. Minibck, and J. W. Kolar, "Generic derivation of dynamic model for half-cycle dcm series resonant converters," IEEE Transactions on Power Electronics, vol. 33, no. 1, pp. 4-7, 2018.

[19] M. Petkovic and D. Dujic, "Hardware-in-the-loop characterization of source-affected output characteristics of cascaded h-bridge converter," IEEE Journal of Emerging and Selected Topics in Power Electronics, vol. 9, no. 3, pp. 3083-3094, 2021 